\title{
THE
}

2-6-2006

\section{Mode Coupling in Quantized High-Quality Films}

\author{
Yiying Cheng \\ University of Rhode Island
}

A. E. Meyerovich

University of Rhode Island, sfo101@uri.edu

Follow this and additional works at: https://digitalcommons.uri.edu/phys_facpubs

Terms of Use

All rights reserved under copyright.

\section{Citation/Publisher Attribution}

Cheng, Y., \& Meyerovich, A. E. (2006). Mode Coupling in Quantized High-Quality Films. Phys. Rev. B, 73(8), 85404. doi: 10.1103/PhysRevB.73.085404

Available at: http://dx.doi.org/10.1103/PhysRevB.73.085404

This Article is brought to you for free and open access by the Physics at DigitalCommons@URI. It has been accepted for inclusion in Physics Faculty Publications by an authorized administrator of DigitalCommons@URI. For more information, please contact digitalcommons-group@uri.edu. 


\title{
Mode coupling in quantized high-quality films
}

\author{
Yiying Cheng and A. E. Meyerovich \\ Department of Physics, University of Rhode Island, 2 Lippitt Rd., Kingston, Rhode Island 02881-0817, USA
}

(Received 23 November 2005; published 6 February 2006)

\begin{abstract}
The effect of coupling of quantized modes on transport and localization in ultrathin films with quantum size effect (QSE) is discussed. The emphasis is on comparison of films with Gaussian, exponential, and power-law long-range behavior of the correlation function of surface, thickness, or bulk fluctuations. For small-size inhomogeneities, the mode coupling is the same for inhomogeneities of all types and the transport coefficients behave in the same way. The mode coupling becomes extremely sensitive to the correlators for large-size inhomogeneities leading to the drastically distinct behavior of the transport coefficients. In high-quality films there is a noticeable difference between the QSE patterns for films with bulk and surface inhomogeneities, which explains why the recently predicted type of QSE with large oscillations of the transport coefficients can be observed mostly in films with surface-driven relaxation. In such films with surface-dominated scattering the higher modes contribute to the transport only as a result of opening of the corresponding mode coupling channels and appear one by one. Mode coupling also explains a much higher transport contribution from the higher modes than it is commonly believed. Possible correlations between the inhomogeneities from the opposite walls provide, because of their oscillating response to the mode quantum numbers, a unique insight into the mode coupling. The presence of inhomogeneities of several sizes leads not to a mechanical mixture of QSE patterns, but to the overall shifting and smoothing of the oscillations. The results can lead to unique non-destructive ways of analysis of the buried interfaces and to study of inhomogeneities on the scales which are inaccessible for scanning techniques.
\end{abstract}

DOI: 10.1103/PhysRevB.73.085404

PACS number(s): 72.10.Fk, 73.23.Ad, 73.50.Bk

\section{INTRODUCTION}

Progress in material technology, especially in nanofabrication, ultrathin film deposition, ultraclean and high vacuum systems, etc., requires better understanding of the effect of remaining bulk inhomogeneities or surface defects on physical processes in high-quality systems. In high-quality systems, these remaining inhomogeneities are small and smooth with a low amplitude and a relatively large lateral scale. In some cases, such as in ultrathin films, the lateral scale of bulk and surface inhomogeneities can even be much larger than the film thickness. Scattering by such small but longrange inhomogeneities is crucial for transport in ultrathin and/or clean systems in which the particle mean free path is comparable to the system size.

Below we consider the effect of random, mostly largescale, bulk, surface, and thickness fluctuations on quantum transport in quantized quasi-two-dimensional (quasi-2D) systems such as quantized flow channels, waveguides, or ultrathin metal films. We will look at the single-particle diffusion coefficient $D$ in a channel as a function of the particle energy and the channel width and at the low-temperature mobility $\mu$ (conductivity $\sigma$ ) as a function of the film thickness and the Fermi wavelength. The main issue is to find how sensitive the transport is to the statistical properties of inhomogeneities, i.e., to the structural or thickness fluctuations with small amplitude and large correlation radius. Here we have in mind large-size surface steps and thickness fluctuations for ultrathin films, slow long-range bending of fibers or films, slowly fluctuating bulk fields, etc. One of the main goals is to separate the effect of the scattering-driven mode coupling from other scattering effects.

The choice of quasi-2D systems is explained by a desire to avoid divergence of surface fluctuations and strong local- ization effects which are inherent to one-dimensional (1D) systems. In contrast to $1 \mathrm{D}$ systems, the randomly fluctuating 2D surfaces are stable while the localization length in systems with weak fluctuations is exponentially large.

Usual approaches to bulk and surface fluctuations are different from each other. The bulk fluctuations are routinely described via the fluctuating bulk potential $V(\mathbf{r})$ or, whenever possible, via the scattering $T$ matrix, $T\left(\mathbf{p}, \mathbf{p}^{\prime}\right)$. Since $\hat{V}$ and $\hat{T}$ are tied to each other via the integral equation,

$$
\hat{T}=\hat{V}+\hat{T} \hat{G} \hat{V}
$$

( $\hat{G}$ is the Green's function), these two descriptions are, in principle, equivalent (and, in the case of weak fluctuations, identical). Below we assume that the bulk inhomogeneities are defined by their scattering $\hat{T}$ matrix and that this $\hat{T}$ matrix is known.

The prevalent way to characterize the surface roughness or thickness fluctuations is to use the correlation function of the surface inhomogeneities

$$
\zeta(\mathbf{s}) \equiv \zeta(|\mathbf{s}|)=\left\langle\xi\left(\mathbf{s}_{1}\right) \xi\left(\mathbf{s}_{1}+\mathbf{s}\right)\right\rangle \equiv A^{-1} \int \xi\left(\mathbf{s}_{1}\right) \xi\left(\mathbf{s}_{1}+\mathbf{s}\right) d \mathbf{s}_{1},
$$

where $\mathbf{s}$ gives the 2D coordinates along the surface, $\xi(\mathbf{s})$ describes the deviation of the position of the surface in the point with $2 \mathrm{D}$ coordinates $\mathbf{s}$ from its average position, $\langle\xi(\mathbf{s})\rangle=0$, and $A$ is the averaging area. This equation assumes that the correlation properties of the surface do not depend on the lateral direction. 
Any transport theory should yield an explicit dependence of the transport coefficients (the particle mean free path, diffusion or mobility coefficients, lateral conductivity, etc.) on the correlator of surface inhomogeneities $\zeta(s)$ and/or the bulk scattering $T$ matrix. Both of these objects can be characterized by their average amplitudes $\ell$, types of decay (exponential, power law, etc.) at large distances or momenta, and the correlation radii $R$ which characterizes this decay. For the surface correlation functions $\zeta$, parameters $\ell$ and $R$ are the average "height" and "lateral size" of surface inhomogeneities or thickness fluctuations. For the bulk scattering $T$ matrix, $\ell$ is the average amplitude in the dependence of the scattering amplitude on dimensionless momentum $p R / \hbar$ and $R$ provides a scale for decay of the scattering amplitude at large momentum transfers. The transport coefficients are determined by the relation between the particle wavelength $\Lambda$, the width of the channel $L$, and the correlation radius of inhomogeneities $R$. If the fluctuations are weak, the fourth length parameter, $\ell$, (more precisely, its square) enters as a coefficient. For example, the conductivity $\sigma$ of degenerate fermions and the single-particle diffusion coefficient $D$ can be parametrized as

$$
\begin{gathered}
\sigma=\frac{2 e^{2}}{\hbar} \frac{R^{2}}{\ell^{2}} f(\Lambda, L, R), \\
D=\frac{\hbar}{m} \frac{R^{2}}{\ell^{2}} d(\Lambda, L, R),
\end{gathered}
$$

with virtually identical functions $f$ and $d$. The reason for this commonality is that both of these transport coefficients are expressed via the same combination of the zeroth and first angular harmonics of the scattering probabilities.

We consider ultrathin systems with quantized motion across the film. Scattering by surface and bulk inhomogeneities could cause coupling of the otherwise distinct quantum modes. We will show that the qualitative behavior of the transport coefficients is extremely sensitive to this scatteringdriven coupling of the modes which, in turn, is determined by the long-range behavior of the structural or thickness fluctuations. Mode coupling has already attracted considerable attention for localization ${ }^{1}$ and transport, especially in the context of applications of the random matrix theory. ${ }^{2}$ Though most of the applications involved transport in systems with bulk disorder, systems with surface disorder were also considered. ${ }^{3-5}$

What has been mostly ignored is the sensitivity of the mode coupling and, in the end, transport, to the type of correlation behavior of disorder. It has been often assumed that the correlation function is short range ( $\delta$-type correlations, hard spheres, etc.) without long tails. What is more, in the case of impurities, a natural assumption is that the correlation radius $R$ ("size") of the disorder is relatively small. Under these assumptions the mode coupling is indeed featureless (though robust) and does not lead to any striking effects which depend on the nature of disorder. However, in highquality quasi-2D samples, it is possible to observe bulk and surface disorder with various correlation properties and fluctuations of different scales. In this case, as we will see below, the mode coupling and transport can follow several distinct scenarios. These different types of behavior are determined by the rate of decay of correlations and their scale and not by the origin of fluctuations or nature and spectra of the particles and waves.

Recently we predicted ${ }^{5}$ a new type of quantum size effect (QSE) with huge, large-period oscillations of conductivity $\sigma(L)$ in films with dominant surface scattering. This surfacedriven effect is so large that a real puzzle is why this different type of QSE has not been observed earlier in high-quality films with bulk scattering. Below we will answer this question by comparing films with bulk and surface scattering. We will also analyze the contribution of different modes to transport. A usual assumption is that in films with surface scattering the main contribution to transport comes form the grazing particles-modes with the lowest quantum numbers. We will see that the scattering-driven mode coupling makes this assumption wrong even for the highest quality films. The last important issue that will be studied below is the possibility of extracting information on the quality of the film surfaces, including the buried ones, from the nondestructive transport measurements. Interestingly, QSE in transport can even reveal a possible correlation between random surface inhomogeneities from the opposite walls.

\section{TRANSPORT IN QUANTIZED SYSTEMS}

Below we deal mostly with the conductivity of metal films and the single-particle diffusion in quasi-2D channels. QSE experiments in metal films include conductivity, ${ }^{6,7}$ spectroscopy, ${ }^{8,9}$ susceptibility, ${ }^{10}$ and $\mathrm{STM}^{11}$ measurements. The signature feature of QSE in metals is a pronounced sawtooth dependence of the lateral conductivity on, for example, film thickness, $\sigma(L)$, common for both bulk ${ }^{12}$ and surface ${ }^{13}$ scattering. However, the QSE experiments in metals have to overcome a difficulty which one does not encounter in semiconductors. The period of the sawtooth QSE oscillations in the dependence $\sigma(L)$ is usually small, nearly atomic, $\hbar / p_{F}$, making the sawtooth behavior of transport almost impossible to observe. For this reason, typical experimental objects are lead or semimetal films such as bismuth. In contrast to this "standard" sawtooth effect, QSE, which is inherent to highquality films, ${ }^{5}$ results in smooth, large-period oscillations of $\sigma(L)$ at relatively large values of $p_{F} L / \hbar$. This QSE could be observed in a wider group of metals. Large-period QSE oscillations have already been observed (see the second Ref. 6); however, the experimental details are still sketchy. This issue is also related to the long-standing controversy on the influence of the structure of the nanoscale film on its resistivity. ${ }^{7}$

Another group of seemingly different physical applications involves the single-particle diffusion in quantized flow channels. Though the typical examples-quantized helium quasiparticles in ultrathin channels ${ }^{14,15}$ and ultracold neutrons in gravitational traps ${ }^{16}$ - seem to be far apart from metal films, the descriptions of the transport processes in such diverse systems are very similar to each other.

Recently, we developed a transparent semianalytical formalism for transport in systems with rough boundaries that 
allows simple uniform calculations in a wide range of systems and for various types of roughness. ${ }^{4,17}$ One attractive feature is that this formalism treats the scattering by surface inhomogeneities using the same transport equation as for the bulk imperfections and even allows one to study the interference between bulk and surface scattering. ${ }^{18}$ This formalism unites earlier approaches by Tesanovic et al. ${ }^{19}$ Fishman and Calecki, ${ }^{20}$ Kawabata, ${ }^{21}$ Meyerovich and S. Stepaniants, ${ }^{22}$ and Makarov et al. ${ }^{23}$ (for a brief review of different theoretical approaches, see Refs. 4 and 24). In this paper we apply this approach to the study of the fluctuation-driven coupling of quantized modes. The limits of applicability of our approach are discussed in detail in Refs. 4 and 18.

Since the 2D mobility of particles is described by essentially the same equations as the exponent in the expression for the localization length in films, our study also provides the dependence of the localization length on the type of the correlation function of random surface and bulk inhomogeneities.

The paper has the following structure. In Sec. III, we introduce the transport equation and expressions for the transport coefficients. Section IV briefly describes various types of the surface inhomogeneities and bulk $T$ matrices used in the computations. The results are given in Sec. V and conclusions in Sec. VI.

\section{TRANSPORT EQUATION AND TRANSPORT COEFFICIENTS}

QSE is caused by quantization of motion in the direction perpendicular to the film, $p_{x} \rightarrow \pi j \hbar / L$, which leads to a split of the energy spectrum $\epsilon(\mathbf{p})$ into a set of minibands, $\epsilon\left(p_{x}, \mathbf{q}\right) \rightarrow \epsilon(\pi j \hbar / L, \mathbf{q})=\epsilon_{j}(\mathbf{q})$. For simplicity, we consider mostly particles with a parabolic spectrum,

$$
\epsilon_{j}(\mathbf{q})=\frac{1}{2 m}\left[(\pi j \hbar / L)^{2}+q^{2}\right] .
$$

The extension to nonparabolic spectra is discussed in Sec. V E. We will look at two similar transport problems, namely, at conductivity of degenerate fermions,

$$
q_{j} \equiv q_{F j}=\left[2 m \epsilon_{F}-(\pi j \hbar / L)^{2}\right]^{1 / 2},
$$

$\left[\epsilon_{F}=\pi^{2} \hbar^{2} / 2 m \Lambda_{F}^{2}\right.$ is the overall Fermi energy, $\Lambda_{F}$ is the Fermi wavelength, and $q_{j}\left(\epsilon_{F}, L\right)$ is the Fermi momentum for the miniband $j$ ] and at single-particle diffusion for particles with energy $E=\pi^{2} \hbar^{2} / 2 m \Lambda^{2}$,

$$
q_{j}=\left[2 m E-(\pi j \hbar / L)^{2}\right]^{1 / 2},
$$

where $q_{j}(E, L)$ is the momentum of such a particle in the miniband $j$. Both problems are computationally similar and reduce to almost identical sets of linear equations. ${ }^{4}$

In the case of conductivity of degenerate fermions, the transport equation for the distribution functions $n_{j}(\mathbf{q})$,

$$
\frac{d n_{j}}{d t}=2 \pi A \sum_{j^{\prime}} \int W_{j j^{\prime}}\left[n_{j^{\prime}}-n_{j}\right] \delta\left(\epsilon_{j \mathbf{q}}-\epsilon_{j^{\prime} \mathbf{q}^{\prime}}\right) \frac{d^{2} q^{\prime}}{(2 \pi \hbar)^{2}},
$$

reduces, after standard transformations, to a set of linear equations

$$
\begin{gathered}
q_{j} / m=-\sum_{j^{\prime}} \nu_{j^{\prime}}\left(q_{j^{\prime}}\right) / \tau_{j j^{\prime}}, \\
\frac{2}{\tau_{j j^{\prime}}}=m \sum_{j^{\prime \prime}}\left[\delta_{j j^{\prime}} W_{j j^{\prime \prime}}^{(0)}-\delta_{j^{\prime} j^{\prime \prime}} W_{j j^{\prime}}^{(1)}\right],
\end{gathered}
$$

where $n_{j}^{(1)}=\nu_{j} \delta\left(\epsilon-\epsilon_{F}\right) e E$ is the first angular harmonic of the distribution function $n_{j}(\mathbf{q})$ at $q=q_{j}$, and $W_{j j^{\prime}}^{(0,1)}\left(q_{j}, q_{j^{\prime}}\right)$ are the zeroth and first harmonics of $W\left(\mathbf{q}_{j}-\mathbf{q}_{j^{\prime}}\right)$ over the angle $\widehat{\mathbf{q}_{j} \mathbf{q}_{j^{\prime}}}$. The solution of Eq. (9) provides the conductivity of the film,

$$
\sigma=-\frac{e^{2}}{3 \hbar^{2}} \sum_{j} \nu_{j}\left(q_{j}\right) q_{j} .
$$

The matrix $\hat{\tau}$ is diagonal when the scattering-driven mode coupling is negligible with respect to the in-band scattering.

The single-particle diffusion is similar to the conductivity problem for degenerate fermions. In equilibrium, a particle with energy $E$ can be in any of $S$ accessible minibands $\epsilon_{j}(\mathbf{q})=(1 / 2 m)\left[(\pi j \hbar / L)^{2}+q^{2}\right]$ for which $\epsilon_{j}(q=0) \leqslant E$. The equilibrium distribution function $n^{(0)}$ is

$$
n^{(0)}(\mathbf{q})=\sum n_{j}^{(0)}(\mathbf{q})=\frac{\pi}{m S} \sum \delta\left[E-\epsilon_{j}(\mathbf{q})\right],
$$

and the transport equation reduces to a set of $S$ coupled linear equations for distributions $\nu_{j}\left(q_{j}\right)$ with momenta $q_{j}(7)$ which is almost identical to Eqs. (9) and (10),

$$
\begin{gathered}
\frac{1}{S m} q_{j} \nabla \rho=-\sum_{j^{\prime}} \nu_{j^{\prime}}\left(q_{j^{\prime}}\right) / \tau_{j j^{\prime}}, \\
D \nabla \rho=-\frac{1}{m} \sum_{j=1}^{S} q_{j} \nu_{j}\left(q_{j}\right),
\end{gathered}
$$

where $\nabla \rho$ is the density gradient that causes the diffusion, $D$ is the single-particle diffusion coefficient, and $\hat{\tau}$ is still defined by Eq. (9). The single-particle mobility coefficient $b$ is related to $D$ by the Einstein equation $D=b E$.

The results for the single-particle diffusion also provide the mean free path $\mathcal{L}$ and the exponent in the expression for the localization length $\mathcal{R}$ that describes localization caused by particle scattering by random wall and/or bulk inhomogeneities, ${ }^{4}$

$$
\mathcal{R}=\mathcal{L} \exp [\pi m S D / \hbar] .
$$

\section{SURFACE CORRELATION FUNCTION VS BULK SCATTERING AMPLITUDE}

In the case of surface or thickness fluctuations, we consider an infinite 2D channel (or film) of the average thickness $L$ with random rough boundaries

$$
x=L / 2-\xi_{1}(y, z), \quad x=-L / 2+\xi_{2}(y, z)
$$

(the walls are assumed hard with infinite potential). The inhomogeneities are small, $\xi_{1,2}(y, z) \ll L$, and random with zero 
average, $\left\langle\xi_{1}\right\rangle=\left\langle\xi_{2}\right\rangle=0$. Their correlation function $\zeta_{i k}(\mathbf{s})$ and its Fourier image $\zeta_{i k}(\mathbf{q})$, which is often called the power spectral density function or, in short, the power spectrum, are defined as

$$
\begin{gathered}
\zeta_{i k}(|\mathbf{s}|)=\left\langle\xi_{i}\left(\mathbf{s}_{1}\right) \xi_{k}\left(\mathbf{s}_{1}+\mathbf{s}\right)\right\rangle \equiv A^{-1} \int \xi_{i}\left(\mathbf{s}_{1}\right) \xi_{k}\left(\mathbf{s}_{1}+\mathbf{s}\right) d \mathbf{s}_{1}, \\
\zeta_{i k}(|\mathbf{q}|)=\int d^{2} s e^{i \mathbf{q} \cdot \mathbf{s}} \zeta_{i k}(|\mathbf{s}|)=2 \pi \int_{0}^{\infty} \zeta_{i k}(s) J_{0}(q s) s d s,
\end{gathered}
$$

where $\mathbf{s}=(y, z)$ and $\mathbf{q}=\left(q_{y}, q_{z}\right)$ are the $2 \mathrm{D}$ vectors. In homogeneous systems, the correlation function depends only on the distance between points $\left|\mathbf{s}_{1}-\mathbf{s}_{2}\right|$ and not on coordinates themselves. The correlation functions $\zeta_{11}$ and $\zeta_{22}$ describe intrawall correlations of inhomogeneities, and $\zeta_{12}=\zeta_{21}$ the interwall correlations. Usually, but not always, the inhomogeneities on different walls are not correlated with each other, $\zeta_{12}=0$. Thus, everywhere, except for Sec. VF, it is assumed that $\zeta_{12}=0$. To avoid parameter clutter, we also assume that the correlation parameters are the same on both walls, $\zeta_{11}=\zeta_{22}=\zeta$. Then the effective correlator contains $2 \zeta(s)$ with $\zeta(s)$ given by equations below.

Surface inhomogeneities exhibit a wide variety of types of the correlation functions. ${ }^{25-28}$ To have a meaningful comparison, we consider the correlation functions that involve only two characteristic parameters, namely, the amplitude (average height) $\ell$ and the correlation radius (average size) $R$ of surface inhomogeneities.

The most commonly used in theoretical applications is the Gaussian correlation function,

$$
\zeta(s)=\ell^{2} \exp \left(-s^{2} / 2 R^{2}\right), \quad \zeta(q)=2 \pi \ell^{2} R^{2} \exp \left(-q^{2} R^{2} / 2\right),
$$

including its limit for small correlation radius $R \rightarrow 0$, i.e., the $\delta$-type correlations,

$$
\zeta(s)=\ell^{2} R^{2} \delta(s) / s, \quad \zeta(q)=2 \pi \ell^{2} R^{2} .
$$

Sometimes, a better fit to experimental data on surface scattering is provided by the use of the exponential correlation function

$$
\zeta(s)=\ell^{2} \exp (-s / R), \quad \zeta(q)=\frac{2 \pi \ell^{2} R^{2}}{\left(1+q^{2} R^{2}\right)^{3 / 2}},
$$

or by the even more long-range, power-law correlators

$$
\zeta(s)=\frac{2 \mu \ell^{2}}{\left(1+s^{2} / R^{2}\right)^{1+\mu}}, \quad \zeta(q)=2 \pi \ell^{2} R^{2} \frac{(q R)^{\mu}}{2^{\mu-1} \Gamma(\mu)} K_{\mu}(q R),
$$

with different values of the parameter $\mu$. The asymptotic behavior of the power spectra, i.e., of the functions $K_{\mu}(q R)$, is essentially exponential. The most commonly used are the Staras function with $\mu=1$ and the correlator with $\mu=1 / 2$ which has the plain exponential power spectrum $\zeta(q)$,

$$
\zeta(q)=2 \pi \ell^{2} R^{2} \exp (-q R) .
$$

The last class of correlation functions covers the powerlaw correlators in momentum space,

$$
\zeta(q)=\frac{2 \pi \ell^{2} R^{2}}{\left(1+q^{2} R^{2}\right)^{1+\lambda}}, \quad \zeta(s)=\ell^{2} \frac{(s / R)^{\lambda}}{2^{\lambda} \Gamma(1+\lambda)} K_{\lambda}(s / R),
$$

which are exponential functions in the configuration space. The correlators from this group include the Lorentzian in momentum space $\lambda=0$ that was observed in Ref. 26 and the exponential correlator (18) at $\lambda=1 / 2$.

The constants in all these correlators are chosen in such a way that the value of $\zeta(q=0)=2 \pi \ell^{2} R^{2}$ is the same. This provides a reasonable basis of comparison for transport coefficients in films with all these different types of random surfaces. Indeed, the scattering cross section for $q \rightarrow 0$ does not depend on the details of short- and mid-range structure of surface inhomogeneities. Therefore, at Fermi momenta $q_{F} \rightarrow 0$ (more precisely, at $q_{F} R \ll 1$ ), the transport coefficients should be the same for all random surfaces. [The only exception is the Lorentzian for which $\zeta(q)$ diverges at small $q$; however, the Lorentzian is nonphysical anyway.] Some useful analytical expressions for the angular harmonics of these correlation functions can be found in Ref. 5 .

To have a uniform comparison of the results throughout the paper, we will plot numerical results for the Gaussian correlator, the power-law correlator in configuration space (19) with $\mu=1$ (the Staras function), and the power-law correlator in momentum space (21) with $\lambda=0.5$ (exponential correlator in real space).

In the case of bulk modulation, it makes sense to start directly from the bulk scattering amplitude $T\left(\mathbf{p}, \mathbf{p}^{\prime}\right)$ which, in the case of quantized films, is transformed into the matrix

$$
T\left(\mathbf{p}, \mathbf{p}^{\prime}\right)=T\left(p_{x}, \mathbf{q} ; p_{x}^{\prime}, \mathbf{q}\right) \rightarrow T_{j j^{\prime}}\left(\mathbf{q}, \mathbf{q}^{\prime}\right)=T\left(\mathbf{q}_{j}-\mathbf{q}_{j^{\prime}}^{\prime}\right)
$$

For this matrix, we also looked at the Gaussian form similar to (16), power-law form similar to (21) with $\lambda=0.5$ (standard Debye screening with an exponent in real space), and exponential form (19) with $\mu=1$. Another interesting possibility here would be an oscillating function which corresponds to an alternative type of screening in metals. However, such a function would require us to introduce two lateral length parameters which would make a meaningful comparison between the correlation functions impossible.

In what follows we compare the transport properties of the films in Eqs. (16)-(21) in a wide range of film thickness $L$, correlation radius $R$, and particle wavelength $\Lambda=\pi / q$ (or the 2D particle density $N$ ).

The transport equation for both bulk and surface imperfections is the same, Eqs. (8)-(12). The only distinguishing feature is the dependence of the scattering probabilities $W_{j j^{\prime}}\left(\mathbf{q}, \mathbf{q}^{\prime}\right)$ on the correlation function of surface fluctuations $\zeta\left(\left|\mathbf{q}-\mathbf{q}^{\prime}\right|\right),{ }^{4,5}$ 


$$
W_{j j^{\prime}}\left(\mathbf{q}, \mathbf{q}^{\prime}\right)=\frac{\hbar}{m^{2} L^{2}}\left[\zeta_{11}+\zeta_{22}+2 \zeta_{12}(-1)^{j+j^{\prime}}\right]\left(\frac{\pi j}{L}\right)^{2}\left(\frac{\pi j^{\prime}}{L}\right)^{2}
$$

and the $T$ matrix for the bulk imperfections,

$$
W_{j j^{\prime}}\left(\mathbf{q}, \mathbf{q}^{\prime}\right)=\frac{2 \pi}{\hbar}\left|T\left(\mathbf{q}_{j}-\mathbf{q}_{j^{\prime}}^{\prime}\right)\right|^{2} .
$$

Note that in contrast to Eq. (23), the dependence of $W$ (24) on band indices $j, j^{\prime}$ is generally not known explicitly and is determined by the $T$ matrix (22). This dependence is the same as in Eq. (23) when the fluctuating bulk field is essentially two-dimensional and can be factorized as

$$
U(x)+(\partial U / \partial x) \xi(\mathbf{s}),
$$

where $x$ and $\mathbf{s}$ are the coordinates across and along the film and $U(x)$ is the field without fluctuations. This situation, though realistic, is by no means general. If, for example, the bulk field fluctuates only along the film and does not change across the film, then $T\left(\mathbf{q}_{j}-\mathbf{q}_{j^{\prime}}^{\prime}\right)=\delta_{j j^{\prime}} T\left(\mathbf{q}-\mathbf{q}^{\prime}\right)$. The offdiagonal elements of $T\left(\mathbf{q}_{j}-\mathbf{q}_{j^{\prime}}\right)$ and, therefore, the mode coupling are associated only with the variation of the bulk field across the film. All this makes modeling of the function $T\left(\mathbf{q}_{j}-\mathbf{q}_{j^{\prime}}\right)$ more ambiguous than for the surface correlator. There are two ways of dealing with this. The first one is to assume that the bulk fluctuations in ultrathin films have the form (25) and, essentially, to use the expression similar to Eq. (23) for the scattering probabilities [of course, without the oscillating interwall term $\zeta_{12}(-1)^{j+j^{\prime}}$. Another approach, which is more appropriate for thicker films, is to assume that the bulk fluctuations are truly three-dimensional and are not affected by the film boundaries except from the straightforward quantization,

$$
T_{j j^{\prime}}\left(\mathbf{q}, \mathbf{q}^{\prime}\right) \equiv T\left(\pi j \hbar / L, \mathbf{q} ; \pi j^{\prime} \hbar / L, \mathbf{q}^{\prime}\right) .
$$

Then, for example, the Gaussian fluctuations in momentum space lead to the following form of $W$ :

$$
\begin{aligned}
W_{j j^{\prime}}\left(\mathbf{q}, \mathbf{q}^{\prime}\right)= & \frac{8 \pi^{5} \hbar^{5} \ell^{2} R^{2}}{m^{2} L^{6}} \exp \left(-q^{2} R^{2} / 2\right) \\
& \times \exp \left[-\frac{\pi^{2}\left(j-j^{\prime}\right)^{2} R^{2}}{2 L^{2}}\right],
\end{aligned}
$$

where we introduced parameters $\ell$ and $R$ in such a way so that to make the description as close as possible to the one with the Gaussian thickness fluctuations (16) [or with the one with the Gaussian bulk fluctuations of the type (25)],

$$
W_{j j^{\prime}}\left(\mathbf{q}, \mathbf{q}^{\prime}\right)=\frac{8 \pi^{5} \hbar^{5} \ell^{2} R^{2}}{m^{2} L^{6}} j^{2} j^{\prime 2} \exp \left(-q^{2} R^{2} / 2\right) .
$$

\section{RESULTS AND DISCUSSION}

\section{A. General comments}

As it is mentioned in the Introduction, the 2D conductivity $\sigma$ of the film has the dimensionality of conductance, $e^{2} / \hbar$.
The conductivity depends on the relation between three length scales-particle (Fermi) wavelength $\Lambda_{F}=1 / q_{F}$, the width of the channel $L$, and the "lateral size" (correlation radius) of inhomogeneities $R$. The fourth length parameter, $\ell$, is perturbative and enters conductivity as a perturbative coefficient $1 / \ell^{2}$,

$$
\sigma=\frac{2 e^{2}}{\hbar} \frac{R^{2}}{\ell^{2}} f\left(L / \Lambda_{F}, R / L\right)
$$

Note, that the conductivity diverges in the limit of vanishing inhomogeneities $\ell \rightarrow 0$ or $R \rightarrow \infty$.

The single-particle diffusion coefficient $D$ can be parametrized in a similar way,

$$
D=\frac{\hbar}{m} \frac{R^{2}}{\ell^{2}} d(L / \Lambda, R / L),
$$

where the dimensionless function $d$ is given by the same combination of the zeroth and first harmonics of the scattering probability $W$,

$$
d(L / \Lambda, R / L)=\frac{3}{2 S} f(L / \Lambda, R / L),
$$

and $S=\operatorname{Int}(L / \Lambda)$ is the number of quantized energy minibands accessible to the particle with the wavelength $\Lambda$. The presence of the step-wise function $S(L / \Lambda)$ in the denominator of Eq. (31) can lead to a visible difference in shapes between functions $d(L / \Lambda, R / L)$ and $f(L / \Lambda, R / L)$ : at large $R / L$ the function $f$ is practically smooth while the function $d$ has residual singularities in the point where the number of accessible minibands changes. These singularities can serve as useful markers that help to separate the "standard" QSE and the QSE of Ref. 5. Therefore, we will plot the results for $d(L / \Lambda, R / L)$ more often than for $f(L / \Lambda)$. Apart from these small-amplitude singularities, the functions $d$ and $f$ are similar.

Below we will plot the dimensionless functions $d(L / \Lambda)$ and $f\left(L / \Lambda_{F}\right)$ at various constant values of $R / L$. The orders of magnitude of these functions change widely depending on the type of surface and bulk correlators and the value of $R / L$. In order to provide a better visual comparison between these functions, we will normalize $d(x)$ and $f(x)$ by their (usually, maximal) values at the maximal value of $x=L / \Lambda$ in the calculation. In other words, we will plot the normalized functions

$$
d(x) / d\left(x_{\max }\right), \quad f(x) / f\left(x_{\max }\right),
$$

where the values of the coefficients $d\left(x_{\max }\right)$ and $f\left(x_{\max }\right)$ are irrelevant for our purposes. In this way, all the functions change from 0 at $x=0$ to 1 at $x=x_{\max }$ and can be visually compared with each other. The values of $x_{\max }$ in our computations vary from 35 to 100 , which means that there are between 35 and 100 quantized minibands accessible to the particles.

The data below involve the Gaussian correlator (16), power-law correlator (19) with $\mu=1$ (the Staras function), and the exponential correlator in real space (18) [i.e., the 


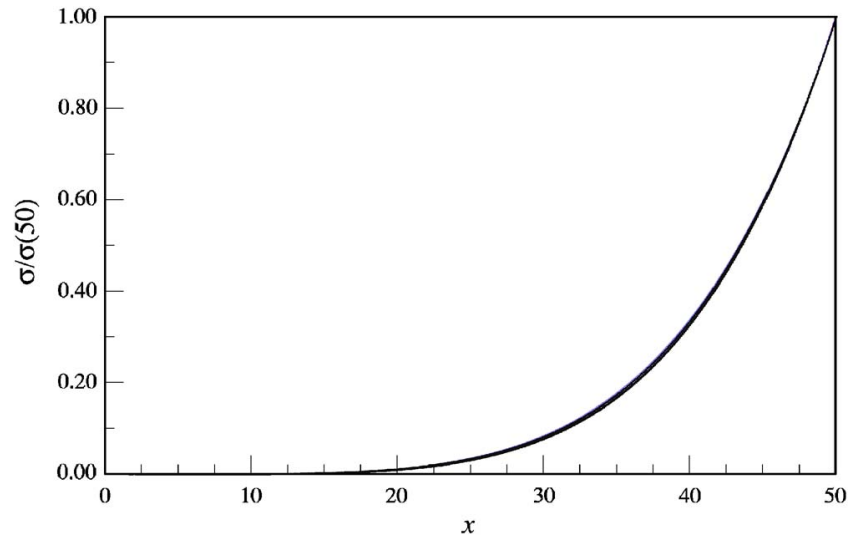

FIG. 1. (Color online) Normalized conductivity $f(x) / f(50)$, $x=L / \Lambda_{F}$, Eq. (29), with artificially frozen mode coupling. The shapes of all five curves are identical irrespective of the type of inhomogeneities (Gaussian, exponential, or power-law) and the value of $R / L$. With this resolution all the curves are smooth.

correlator (21) with $\lambda=0.5]$. We will refer to the latter two as the power-law and exponential correlators without specifying $\mu$ and $\lambda$.

\section{B. Mode coupling}

To underscore the effects of mode coupling, we start from the calculation with the artificially frozen mode coupling (the off-diagonal components of the matrix of scattering probabilities $W_{j j^{\prime}}$ are not calculated, but made equal to zero, $\left.W_{j j^{\prime}}=W_{j} \delta_{j j^{\prime}}\right)$. This is a good starting point since in highquality films with $R / L \gg 1$ the mode coupling is suppressed anyway. In this case the transport equations (9) and (12) can be solved analytically, ${ }^{17}$

$$
\sigma \simeq \frac{2 e^{2}}{3 \hbar^{2} m^{2}} \sum_{j} \frac{q_{j}^{2}}{W_{j}^{(0)}-W_{j}^{(1)}},
$$

and similarly for diffusion,

$$
D \simeq \frac{1}{m^{3} S} \sum_{j} \frac{q_{j}^{2}}{W_{j}^{(0)}-W_{j}^{(1)}},
$$

where $W_{j}^{(0,1)}$ are the zeroth and first angular harmonics of the transition probabilities (23) and (24). Note, that since $W_{j}$ for surface correlators grows proportionally to $j^{4}$, Eq. (23), the sum in Eq. (33) is rapidly convergent and the contribution of the higher modes in systems without mode coupling can be negligible. This means that for the surface scattering without mode coupling the dependence of the conductivity $\sigma$ on the film thickness is practically a smooth function, Fig. 1 (small kinks on the curves cannot be seen with the resolution of the figure). This is not so for the single-particle diffusion (34) which contains a step-wise factor $S(L)$, Fig. 2. The small sawtooth drops on the curves $d(L)$ at the points in which $S=\operatorname{Int}(L / \Lambda)$ changes by 1 (quantum size effect, QSE) can serve as useful markers on the curves that help to separate the standard QSE from other phenomena. Therefore, more often than not we will plot $d(L)$ rather than $\sigma(L)$. Figures 1

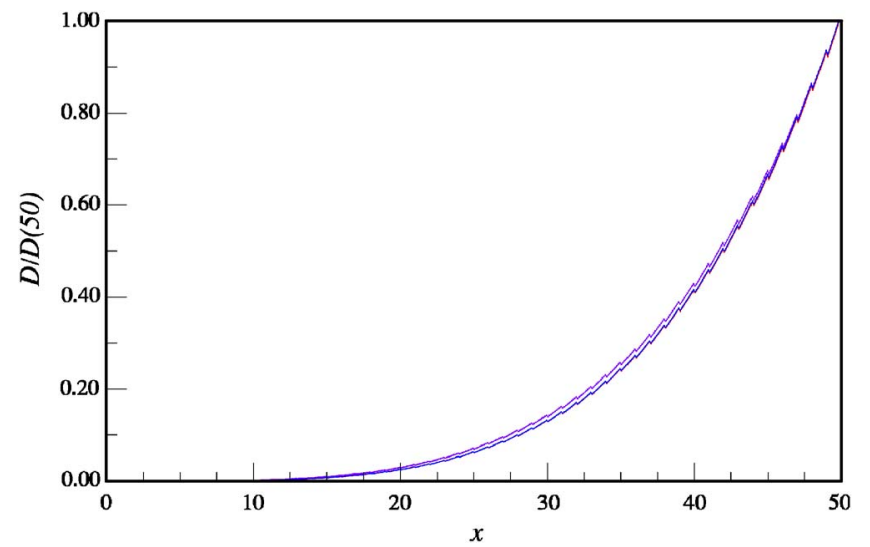

FIG. 2. (Color online) Normalized single-particle diffusion coefficient $d(x) / d(50), x=L / \Lambda$ with artificially frozen mode coupling. The shapes of all five curves are identical irrespective of the type of inhomogeneities (Gaussian, exponential, or power law) and the value of $R / L$. Small sawtooth anomalies correspond to changes in the number of accessible minibands $S(L)$, Eq. (31).

and 2 combine the data for the Gaussian, exponential, and power-law inhomogeneities and five different values of $R / L=0.1,1,10,50,100$. All normalized curves $f(x) / f(50)$ and $d(x) / d(50)$ are identical to each other except, of course, for the normalization coefficients $f(50)$ and $d(50)$, which change by the orders of magnitude depending on $R / L$ and the type of inhomogeneities.

\section{Bulk vs surface scattering}

In Ref. 5 we reported the existence of a class of QSE for a "boring" type of high-quality films $R \gg L$ with Gaussian or exponential (in momentum space) surface and thickness fluctuations. This QSE manifests itself as giant oscillations of $\sigma(L)$ with a relatively large period which is directly related to the correlation radius of surface inhomogeneities,

$$
L_{j} \simeq \frac{\pi}{2} \sqrt{(2 j+1) R \Lambda}
$$

where $L_{j}$ are the positions of the peaks in $\sigma(L)$.

The effect is so large and well pronounced that a natural question is why has it not been observed earlier, for example, in impurity scattering. As we discussed in the Introduction and Sec. IV, the equations that describe surface and bulk scattering are so similar that it looks like this different QSE should be observed in bulk scattering as well. Surprisingly, this is not the case. Figure 3 presents the normalized diffusion coefficient for surface and bulk scattering. Both surface and bulk correlation functions are Gaussian with the same large value of $R / L=50$. The curve with surface scattering exhibits large QSE oscillations with peaks in positions (35), while the bulk scattering results in a monotonic dependence similar to that for systems with frozen mode coupling in Figs. 1 and 2. This surprising difference between the bulk and boundary scattering requires an explanation.

Our explanation of the "new" QSE, Eq. (35), in Ref. 5 was that in high-quality films with $R / L \gg 1$ the mode cou- 


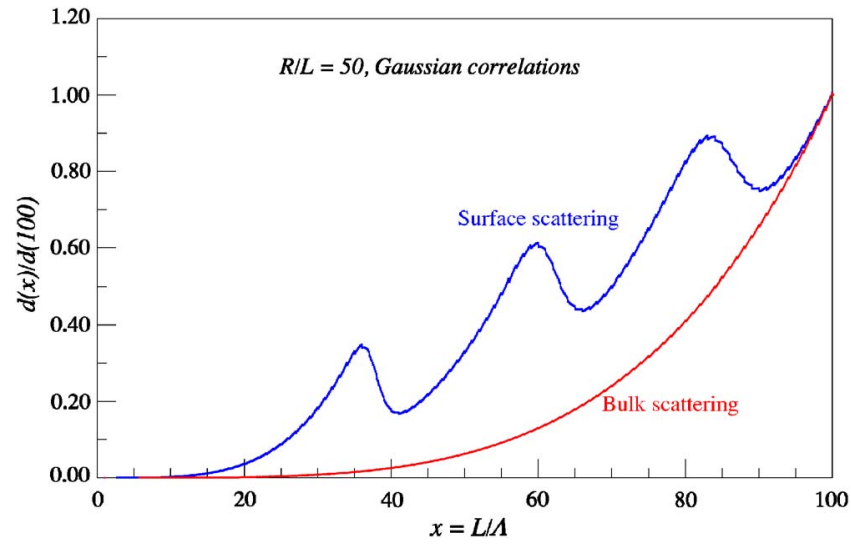

FIG. 3. (Color online) Normalized single-particle diffusion coefficient with surface and bulk scattering; in both cases the inhomogeneities are Gaussian with $R / L=50$.

pling is largely suppressed because the scattering-driven change in momentum $\delta q \sim 1 / R$ is insufficient to induce transitions between the modes which are widely separated between themselves at small $L, \delta \epsilon \propto 1 / L^{2}$. The mode coupling processes turn on one by one only at the values of thickness given by Eq. (35). This should be the same for both surface and bulk scattering. What is not the same is the effect that this opening of the mode coupling channels has on transport. In the case of surface scattering, the modes with the lowest quantum numbers (grazing particles) make the largest contribution to transport (without the quantum cutoff, the contribution from the particles in the lowest mode-grazing particles moving parallel to the surface-would have been infinite). Therefore, the threshold opening of individual mode coupling channels for the lowest modes, which almost doubles the corresponding cross sections, is very noticeable in transport. In the case of bulk scattering, the situation is different. Here all modes contribute more or less equally to transport. Therefore, in the case of a large number of available modes, the opening of few mode-coupling channels in points (35) is unnoticeable and the transport coefficients remain nearly the same as in the pure diagonal case. This explains why this different QSE (35) had not been discovered earlier when studying the bulk-dominated transport.

Figure 4 illustrates very different sensitivity of the surface- and bulk-driven transport to mode coupling. The figure contains the normalized single-particle diffusion coefficient in the cases of bulk and surface scattering. In both cases the inhomogeneities are Gaussian with four different values $R / L=0.1,1,10,50$. In the case of surface scattering, the pattern evolves from the "usual" QSE sawtooth curve for $R / L=0.1$ to the different QSE with huge oscillations on more or less smooth curve for $R / L=50$ (the curves are marked by the value of $R / L$ ). In the case of bulk scattering, all the curves split into two groups of smooth curves for small and large $R / L$ (with, correspondingly, robust and mostly suppressed mode coupling). Though the shapes of the curves from these two groups are distinctly different, the difference in shapes, in contrast to surface scattering, is rather quantitative than qualitative. Of course, the sawtooth anomalies, which are inherent to QSE, are more distinct on the curves with robust mode coupling at small $R / L$.

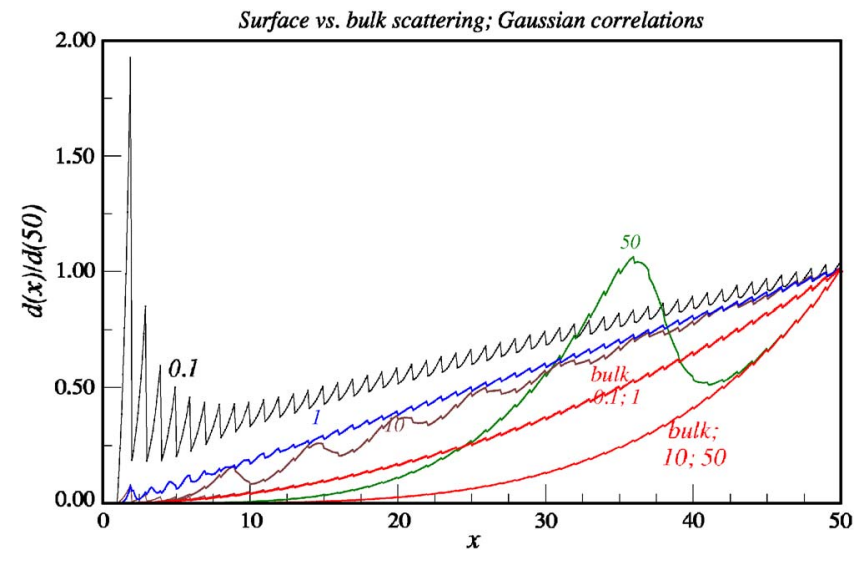

FIG. 4. (Color online) Normalized single-particle diffusion coefficient with surface and bulk scattering; in both cases the inhomogeneities are Gaussian with $R / L=0.1,1,10,50$. For surface scattering, all four curves, marked by the values of $R / L$, are different. For bulk scattering, there are two groups of coinciding curves with small and large $R / L$.

Summarizing, QSE and the manifestations of the mode coupling are distinctly different in surface- and bulkdominated quantized systems. This is not due to the difference in mode coupling processes themselves, but due to the different patterns for contributions from individual modes, especially for the grazing particles. This also answers a puzzling question why the different type of QSE is observed primarily in systems with the dominant surface relaxation.

\section{Opening of mode coupling channels and mode contributions for surface scattering}

The next step is the analysis of contributions from individual modes and from the mode coupling transitions. We start from the data for the artificially frozen mode coupling transitions in Figs. 1 and 2 for surface scattering and turn on such transitions. The results strongly depend on the size of inhomogeneities $R / L$. Analysis of the scattering probabilities $W$ shows that for all reasonable types of inhomogeneities the decay of the surface-driven scattering probabilities $W_{i j^{\prime}}$ at large $j, j^{\prime}$ is a function of $\left(j+j^{\prime}\right)^{2} R^{2} / L^{2}$ and $\left(j-j^{\prime}\right)^{2} R^{2} / L^{2}$. Therefore, for small inhomogeneities, $R / L \ll 1$, the the mode-coupling scattering probabilities $W_{j j^{\prime}}$ with $j^{\prime} \neq j$ have the same order of magnitude as for the intraband scattering $W_{j j}$. What is more, the contributions of the higher modes to particle transport are quite noticeable and decrease rather slowly with increasing $j, j^{\prime}$. This is illustrated in Fig. 5 for the power-law inhomogeneities $(R / L=0.1)$ where three curves correspond to diffusion in the single-mode, twomode, and three-mode regimes including the mode coupling. The surface correlator is exponential in momentum space, Eq. (19), with $\mu=1$ (the Staras correlator). All three curves are normalized by the single value of $d(50)$ for the singlemode curve. As one can see from the figure, the turning on of the mode coupling does not lead to any qualitative changes and results simply in the increase of the overall scattering cross section. The contributions from all modes have the same order of magnitude. 


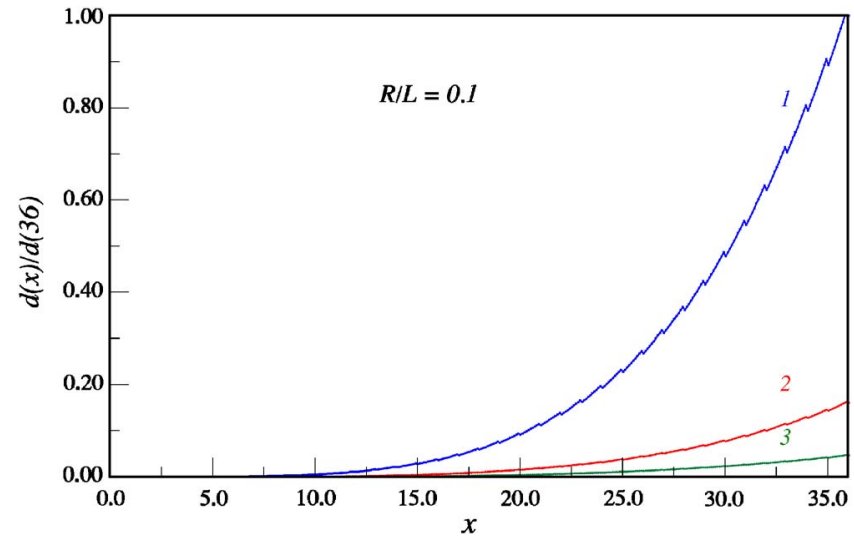

FIG. 5. (Color online) Normalized single-particle diffusion coefficient $d(x) / d(36), x=L / \Lambda$ for power-law inhomogeneities, Eq. (19) at $\mu=1$ for small size inhomogeneities, $R / L=0.1$. Curve 1 takes into account only the main mode. Curve 2 accounts for the first two modes, including coupling; curve 3 , the first three modes. It is clear that all three modes are equally important. All three curves use the same normalization parameter $d(36)$ taken from the single (main) mode contribution (curve 1).

The situation changes dramatically at large $R / L$. Figure 6 presents the same calculation as for Fig. 5 but for $R / L=100$. As one can see, at small $x=L / \Lambda$ all three curves coincide. This means that the contribution of higher modes and the mode-coupling effects are negligible even though the interband transitions are allowed. This is explained by the fact that in high-quality films with $R / L \gg 1$ the modecoupling transitions switch on one by one when the values of the film thickness reaches the values (35) [with a small logarithmic correction which depends on the correlation function of inhomogeneities; Eq. (35) assumes that the quantum numbers $j$ are small and $\left.q_{j} \sim \hbar / \Lambda\right]$. Therefore, at thicknesses $L<L_{1}$ (the first branching point in the curves) all interband transitions $j \leftrightarrow j^{\prime}$ are suppressed. Since at $R / L \gg 1$ the absolute values of $(j R / L)^{2}$ grow very rapidly with $j$, the contributions of the higher modes are negligible as well, and the

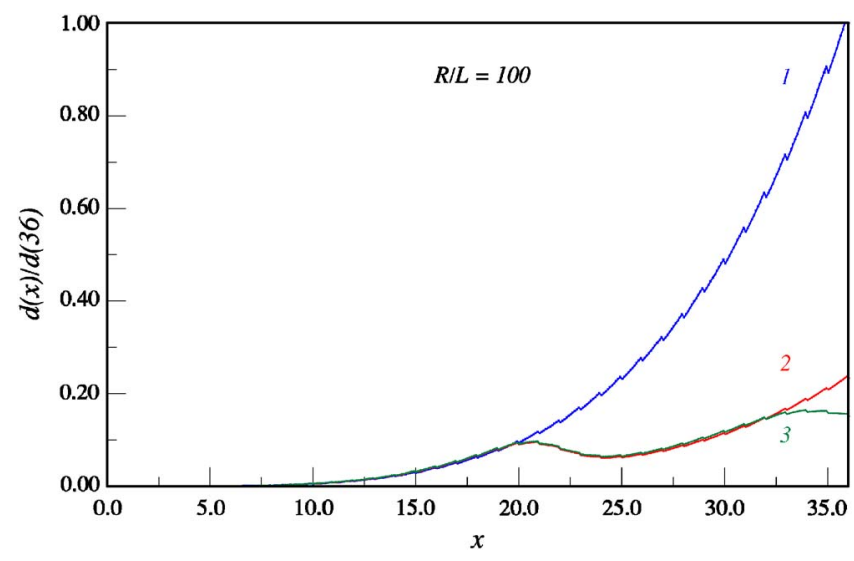

FIG. 6. (Color online) The same as Fig. 5 but for large-scale inhomogeneities, $R / L=100$. The splits occur in the points when the mode coupling becomes noticeable. It is clear that the contributions of the higher modes become noticeable only when their coupling to the main mode becomes large. system is effectively in a single-mode regime. At $L \sim L_{1}$, Eq. (35), the mode-coupling probability $W_{12}$ becomes comparable to $W_{11}$ and the mode-coupling between the two lowest modes becomes robust. Only at this point does the contribution of the second mode become noticeable. Therefore, at $L_{1}<L<L_{2}$ the system is effectively in a two-mode regime, at $L_{2}<L<L_{3}$-in a three-mode regime, and so on, as it is seen clearly in Fig. 6.

The results for the Gaussian correlator are similar to those for the power-law one. The exponential correlators, on the other hand, do not exhibit behavior similar to Fig. 6. The power spectrum for such correlators is decaying very slowly at large $q$ thus ensuring robust mode-coupling transitions at any $R / L$. As a result, the curves $d(L)$ and $\sigma(L)$ resemble those in Fig. 5 at any value of $R / L$.

The important conclusion here is that in high-quality films $R / L \gg 1$ with the surface-driven scattering the contribution of the higher modes becomes important only as a result of the emergence of mode coupling at the values of the film thickness (35). However, after the mode coupling turns on at certain values of the film thickness, the contribution of the higher mode becomes much bigger than it is usually believed.

\section{E. Particles with nonquadratic energy spectrum}

It is very interesting to check to what extent our analysis of the mode coupling effects is sensitive to the form of particle spectra. We start from deriving an analog of Eq. (35) for nonparabolic particles, i.e., from finding the critical values of thickness at which the mode coupling become noticeable for individual modes in high-quality films, $R / L \gg 1$.

Let us consider particles with a bulk spectrum $\epsilon(\mathbf{p})$, or, after quantization, $\boldsymbol{\epsilon}_{j}(\mathbf{q})=\epsilon(\pi \hbar j / L, \mathbf{q})$. Scattering by inhomogeneities of the lateral size $R$ change the lateral momentum by $\delta q \sim \hbar / R$. This small change in momentum $(R \gg L)$ is sufficient for the interband transition $j \leftrightarrow j+1$ only if the energy conservation, $\epsilon_{j}(\mathbf{q})=\epsilon_{j+1}(\mathbf{q}-\hbar / R)$, can be satisfied

$$
0=\frac{\partial \epsilon_{j}}{\partial j}-\frac{\hbar}{R} \frac{\partial \epsilon_{j}}{\partial q} .
$$

The solution of this equation gives the values of the critical thickness $L_{j}$ at which the mode coupling channels $j \leftrightarrow j+1$ become open. [A more accurate equation than (36) contains coefficients of the order of 1 which depend on detail of the correlation function of inhomogeneities.] In the case of parabolic inhomogeneities, as for all particles for which the energy spectrum $\boldsymbol{\epsilon}(\mathbf{p})$ depends only on the absolute value of momentum $p$, Eq. (36) reduces to (35).

As an example, we consider "ultrarelativistic" particles, $\epsilon=c p$, i.e.,

$$
\boldsymbol{\epsilon}_{j}(\mathbf{q})=c \sqrt{(\pi \hbar j / L)^{2}+q^{2}} .
$$

The applications include photons between two (rough) mirrors or quantized phonons in helium films. For nonparabolic spectra $\epsilon(p)$, Eq. (23) for the scattering probabilities $W_{j j^{\prime}}$ should be modified as ${ }^{4}$ 


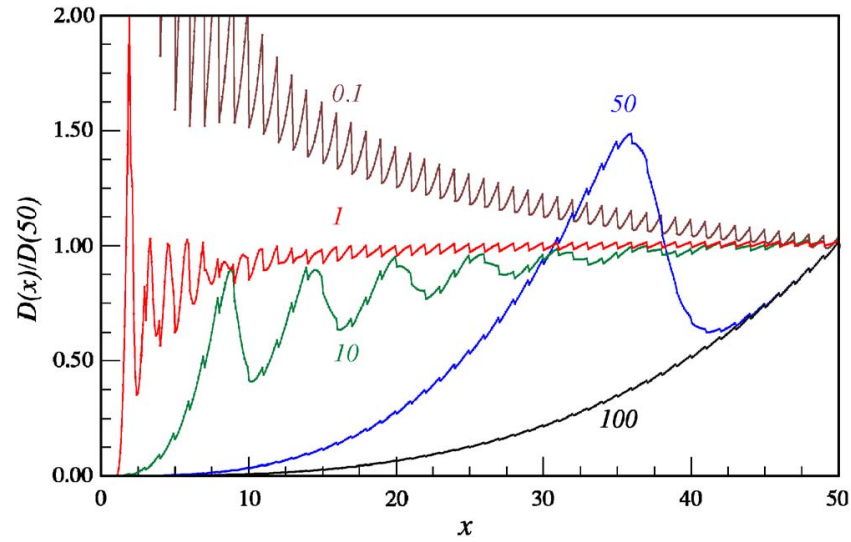

FIG. 7. (Color online) Normalized diffusion coefficient $D(x) / D(50)$, Eq. (39), for ultrarelativistic particles, $\epsilon=c p$, and random inhomogeneities with the Gaussian correlation function. The curves are marked by the values of $R / L ; x=\omega L / \pi c$ is the dimensionless energy (frequency).

$$
\begin{aligned}
W_{j j^{\prime}}\left(\mathbf{q}, \mathbf{q}^{\prime}\right) \delta\left(\epsilon_{j \mathbf{q}}-\epsilon_{j^{\prime} \mathbf{q}^{\prime}}\right) \\
=\frac{j^{2} j^{\prime 2}}{L^{2} \hbar^{3}} \frac{\left(\epsilon_{j^{\prime} \mathbf{q}}-\epsilon_{j \mathbf{q}^{\prime}}\right)^{2}}{\left(j^{2}-j^{\prime 2}\right)^{2}}\left[\zeta_{11}+\zeta_{22}+2 \zeta_{12}(-1)^{j+j^{\prime}}\right] \\
\quad \times \delta\left(\epsilon_{j \mathbf{q}}-\boldsymbol{\epsilon}_{j^{\prime} \mathbf{q}^{\prime}}\right),
\end{aligned}
$$

while $q_{j} / m$ in the equations for the single-particle diffusion (12) should be replaced by the lateral velocity $v_{j}=\partial \epsilon_{j} / \partial q$. Then straightforward algebra leads to the following expression to the single-particle diffusion coefficient:

$$
\begin{gathered}
D=\frac{c R^{3}}{\ell^{2}} d(\omega L / \pi c), \\
d(x)=\frac{1}{2 \pi^{6} x^{3} S(x)}\left(\frac{R}{L}\right)^{3}\left(\frac{q_{j} R}{\hbar}\right) \widetilde{\tau_{j j^{\prime}}}-1\left(\frac{q_{j^{\prime}} R}{\hbar}\right),
\end{gathered}
$$

where $\widetilde{\tau_{j j^{\prime}}}{ }^{-1}$ is the dimensionless inverse relaxation time matrix (9) with the changes mentioned above.

Figure 7 presents normalized diffusion coefficient $D(x) / D(50)$, Eq. (39), for random inhomogeneities with the Gaussian correlation function; $x=\omega L / \pi c$ is the dimensionless energy (frequency). The curves are marked by the values of $R / L$. The curves with small $R / L$ exhibit the "standard" sawtooth QSE. The curves for larger inhomogeneities exhibit the large-scale oscillations with the peaks which correspond to opening of the mode-coupling channels and which are described approximately by Eq. (36). The curves for the exponential in momentum space surface correlators (19) are, essentially, the same. If the surface correlator has a powerlaw shape in momentum space, which means that there exist inhomogeneities of all sizes, the transport coefficients for particles with nonquadratic spectra assume the same "standard" sawtooth shape as for the particles with a parabolic spectrum.

The general conclusion is that the mode coupling effects, which manifest themselves in this type of QSE in transport in high-quality films, are very robust and are not sensitive to the type of the energy spectrum. This means that this type of QSE should exist for (quasi-)particles of different nature and for various solid state systems.

\section{F. Interwall interference effects}

What also makes the scattering by surface inhomogeneities different from scattering by bulk fluctuations or impurities is the possible interference of particle reflected from the opposite wall. This interference is especially interesting in the case when the inhomogeneities from the opposite walls are correlated. In this section we discuss the effect of this interwall correlation of inhomogeneities on mode coupling. The existence of this nontrivial effect is a unique feature of surface scattering that does not have any analog in scattering by bulk inhomogeneities. Surprisingly, the possibility of cross-wall correlation of surface inhomogeneities from opposite walls gives an interesting insight into mode coupling. The study of the effect of interwall correlation of inhomogeneities has been initiated in Refs. 5, 17, and 18 (for additional results in application to excitons see Refs. 29).

The effect of interwall correlations has two unique features stemming from the sign of the interference of scattering from opposite walls. Because of the $(-1)^{j+j^{\prime}}$ factor in the interwall contribution to the scattering probability, Eq. (23), the contribution of the interwall correlation function $\zeta_{12}$ has different signs for in-band $\left(j=j^{\prime}\right)$ and mode-coupling $\left(j=j^{\prime} \pm 1\right)$ scattering processes. Depending on the magnitude of $\zeta_{12}$ and its sign, its contribution can enhance or suppress the mode coupling effects.

To decrease the number of parameters, we assume that, as in Refs. 5, 17, and 18, the correlation functions of inhomogeneities on both walls $\zeta_{11}$ and $\zeta_{22}$ are given by the same function, $\zeta_{11}(s)=\zeta_{22}(s)=\zeta(s)$. The structure of the interwall correlator of inhomogeneities $\zeta_{12}(s)$ is assumed to be the same as for the intrawall correlations with the same correlation radius $R$. However, the amplitude $a$ of the interwall correlations is different from the intrawall ones,

$$
\zeta_{11}=\zeta_{22}=\zeta(s), \quad \zeta_{12}(s)=a \zeta(s), \quad|a| \leqslant 1 .
$$

Note that in contrast to the on-wall correlation functions $\zeta_{11}, \zeta_{22}$, the sign of the interwall correlation function $\zeta_{12}$ is not fixed; even $\zeta_{12}(s=0)$ can be negative. By itself, the sign of the interwall correlations $\zeta_{12}=\left\langle\xi_{1} \cdot \xi_{2}\right\rangle$ is ambiguous and depends on how we introduce the signs of the deviations of the wall positions $\xi_{1,2}$ from the averages $\pm L / 2$; throughout this paper, we use the definitions (14). With this definition, the sign of $a$ can be positive or negative depending on whether the inhomogeneities from the opposite walls "attract" or "repel" each other. If the inhomogeneities from the opposite walls simply reproduce each other ("parallel" walls; the film thickness is constant along the film), then, with our definition of the wall inhomogeneities Eq. (14), $a=-1$. This type of interwall correlation is likely to occur when an ultrathin film grows on an inhomogeneous substrate. In the opposite case of walls with opposite modulations ("antiparallel" walls), $a=1$. This is the case of pure thickness fluctuations, which is likely to occur, for example, after the film (wire) has been inhomogeneously stretched. In the case of parallel 


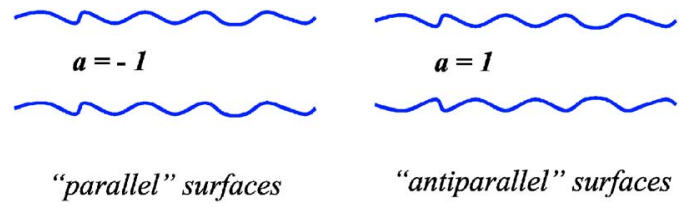

FIG. 8. (Color online) Two different types of walls with correlated random inhomogeneities. For "parallel" walls the interwall correlation amplitude $a=-1$, for "antiparallel" walls, $a=1$. In general, the interwall correlation amplitude $-1 \leqslant a \leqslant 1$.

walls, $a \rightarrow-1$, the destructive interference of scattering by opposite walls can, in the absence of mode coupling (see below), completely negate all transport manifestations of the wall corrugation. These two limiting situations is presented in Fig. 8. In general, $-1 \leqslant a \leqslant 1$.

To extract the effect of interwall correlations, we will present the results for the transport coefficient at different values of the interwall amplitude $a$ and calculate the relative change of conductivity $\sigma$ and diffusion coefficient $D$ caused by the introduction of such correlations,

$$
\phi^{(a)}=\frac{f^{(a)}-f^{(0)}}{f^{(0)}}, \quad \frac{d^{(a)}-d^{(0)}}{d^{(0)}},
$$

where $f^{(a)}$ and $f^{(0)}\left(d^{(a)}\right.$ and $\left.d^{(0)}\right)$ are the values of $\sigma$ and $D$, Eqs. (29) and (30), calculated with and without interwall correlations. An additional benefit of this definition is that the functions $\phi^{(a)}$ are automatically normalized.

In the presence of interwall correlations (40), the transition probabilities $W_{j j^{\prime}}\left(\mathbf{q}, \mathbf{q}^{\prime}\right)$ (23) become proportional to

$$
2\left[1+a(-1)^{j+j^{\prime}}\right] \zeta\left(\left|\mathbf{q}_{j}-\mathbf{q}_{j^{\prime}}^{\prime}\right|\right) .
$$

The most interesting effects of the interwall correlations are related to the oscillating structure of the term with $a$ in Eq. (42).

If the mode coupling is suppressed, then the only important terms in Eq. (42) are the diagonal ones with $j=j^{\prime}$ and the function $\phi^{(a)}$ is a constant,

$$
\phi^{(a)}[\text { no mode coupling }]=\frac{1}{1+a}-1=\frac{-a}{1+a} .
$$

In this case the presence of interwall correlations leads to a simple increase or decrease, depending on the sign of $a$, of the transport coefficients by the factor $-a /(1+a)$. This is always the case, for example, when only one mode is important. ${ }^{29}$ Therefore, all deviations of $\phi^{(a)}$ from the constant (42) are due solely to the scattering-driven mode coupling. This gives a nontrivial insight into the mode coupling and its consequences.

For example, as it is clear from Fig. 6, the mode coupling for the power-law inhomogeneities with $R / L=100$ appears only at $x=L / \Lambda>15$. Therefore, the function $\phi^{(a)}(x<15)$ should be flat, Eq. (43), and exhibit anomalies in the points in which the mode coupling effects are switched on. This is illustrated in Fig. 9 which presents the function $\phi^{(a)}(x)$ for the same power-law inhomogeneities as Fig. $4(R / L=100)$ at five different values of $a, a=-0.5,-0.1,0.1,0.5,09$. The flat parts on left-hand side of all curves $(x<15)$ correspond to

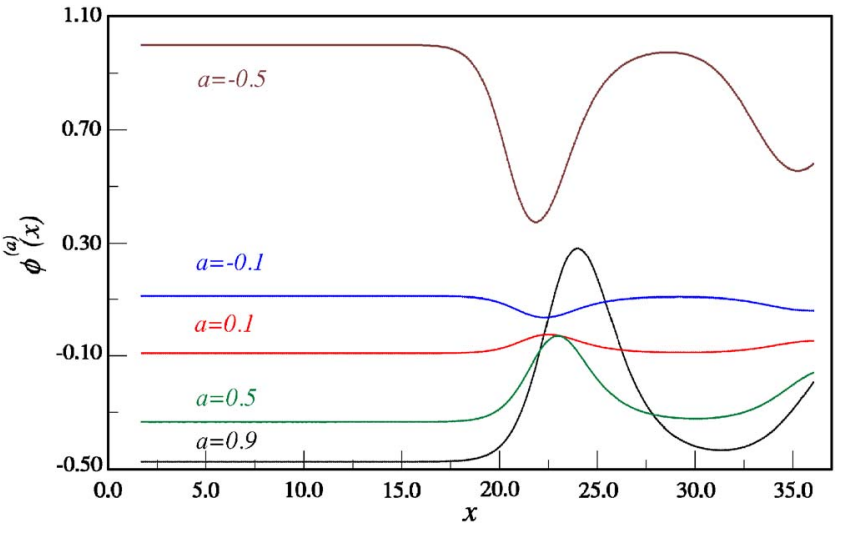

FIG. 9. (Color online) Relative contribution of the interwall correlations to the single-particle diffusion, Eq. (41). Large-size $(R / L=100)$ power-law inhomogeneities, Eq. (19) with $\mu=1$. The curves are labeled by the values of the interwall amplitude $a$.

the absence of mode coupling at these values of $R / L$ and are given by Eq. (43). The peaks in the curves show the values of $x=L / \Lambda$ for the consecutive openings of the mode scattering channels. The difference in the amplitudes of the peaks is easily explained by the dependence of the scattering probabilities on the interwall correlation amplitude $a$, Eq. (42).

The contribution of the term with $a$ in (42) has a different sign for different $W_{j j^{\prime}}$ depending on whether $j+j^{\prime}$ is even or odd. Since the mode coupling channels $j \leftrightarrow j+1$ in highquality films with Gaussian and exponential power functions turn on one by one with increasing $L$, one would expect that the function $\phi^{(a)}(L)$ in such films should become a stepwise function of $L$. This is not correct. In films with large $R / L$ and frozen out mode coupling effects, the contributions of individual modes decrease rapidly as $1 / j^{4}$ (Ref. 17). However, when the transitions $j \leftrightarrow j+1$ are switched on, the overall contribution of the mode $j+1$ increases disproportionately (Fig. 6). As a result, the function $\phi^{(a)}$ becomes an oscillating rather than stepwise function as it is seen clearly in Fig. 9.

The positions of the QSE peaks in systems with interwall correlations differ from Eq. (35) and depend on the value of $a$. The shifts of peaks in Fig. 9, which depend on the value of $a$, are better illustrated in Fig. 10 in which we presented the normalized diffusion coefficient itself (and not the function $\left.\phi^{(a)}\right)$ for the same type of surface inhomogeneities and the same value of $R / L=100$ for two different interwall amplitudes, $a=-0.9,0.9$ as a function of $x=L / \Lambda$. The explanation of these shifts is the following. Let us assume that the first peak is observed at $x=x_{1}$. In this point the value of $W_{12}(x)$ reaches $W_{11}(x), W_{11}\left(x_{1}\right)=W_{12}\left(x_{1}\right)$. According to Eq. (42) in the presence of interwall correlations, these scattering probabilities $W^{(a)}$ change with respect to their values $W^{(0)}$ in the absence of interwall correlations as

$$
W_{11}^{(a)}=(1+a) W_{11}^{(0)}, \quad W_{12}^{(a)}=(1-a) W_{12}^{(0)} .
$$

Since near the peak position $W_{12}^{(0)}(x)$ grows very rapidly while $W_{11}^{(0)}$ does not change much, $W_{12}^{(a)}(x)$ reaches the value $W_{11}^{(a)}$ earlier than $W_{12}^{(0)}(x)$ reaches $W_{11}^{(0)}$ at negative $a$ and later at positive $a$. This exactly what is happening in Fig. 10. At 


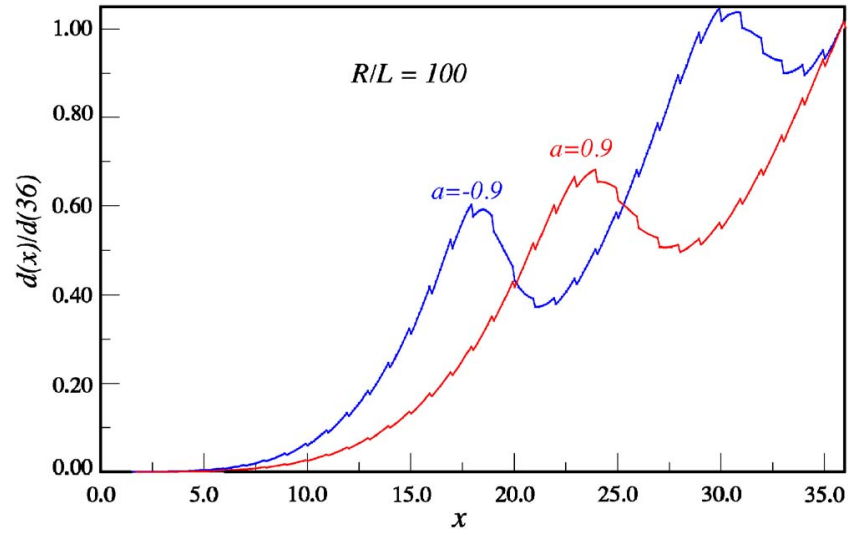

FIG. 10. (Color online) Normalized single-particle diffusion coefficient $d(x) / d(36)$ for the same inhomogeneities as in Fig. 8 for two values of the interwall amplitude $a, a=-0.9,0.9$.

small $a$, the change in position $x_{1}$ with respect to its value in the absence of interwall correlations is

$$
\Delta x_{1}=2 a W_{11}^{(0)}\left(x_{1}\right) /\left[\partial W_{12}^{(0)} / \partial x-\partial W_{11}^{(0)} / \partial x\right] .
$$

The oscillating nature of the interwall contribution, Eq. (42) should be more pronounced for the systems with smaller inhomogeneities, in which the mode-coupling transitions are as probable as the intraband scattering. In this case the flat areas (43) should be absent. Instead, the curves $\phi^{(a)}(x)$ should exhibit QSE with the oscillations in points $x_{S}$ in which the number of occupied minibands $S$ changes by 1 , $S \rightarrow S+1$. (With our choice of dimensionless variables, the period of these oscillations is equal to 1.) This is illustrated in Fig. 11 and 12 which contain the data similar to those in Fig. 9 but for smaller inhomogeneities, $R / L=0.1$ and $R / L=1$ respectively. At $R / L \ll 1$, when all mode coupling transitions and intraband scattering are equally probable, the addition of an extra band $S$ adds all interwall terms with the sign-changing coefficients $a(-1)^{j+S}$, Eq. (42). However, since the main mode, $j=1$, contributes the most to the transport flow, the overall sign of the interwall contribution is the sign of $a(-1)^{1+S}$ and should change in the points in which $S(x)$

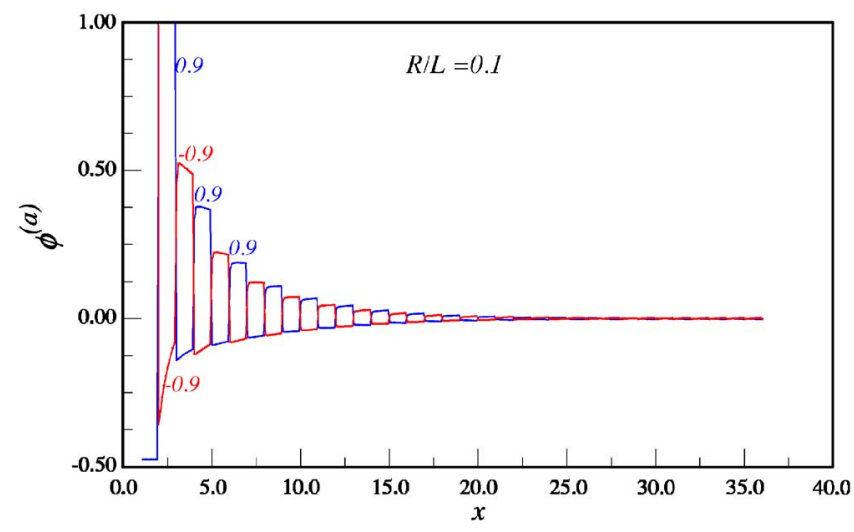

FIG. 11. (Color online) The same as in Fig. 8 but for small inhomogeneities, $R / L=0.1$. The curves are labeled by the values of the interwall amplitude $a=-0.9,0.9$.

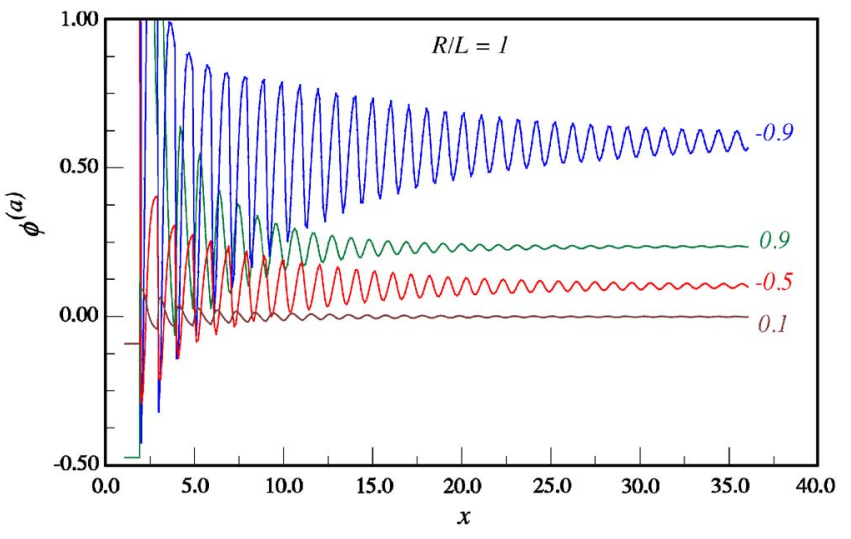

FIG. 12. (Color online) The same as in Fig. 8 but with $R / L=1$. The curves are labeled by the values of the interwall amplitude $a$.

changes. This is exactly what can be seen in Fig. 11. The amplitude of the oscillations grows with an increase in $|a|$ and goes down with increasing $x$. Figure $11(R / L=0.1)$ demonstrates these oscillations for large interwall correlations, $a=-0.9,0.9$. Since the signs of these two interwall amplitudes are opposite, the contributions from these two types of cross-correlations have opposite signs, Eq. (42). The analogous curves for all interwall amplitudes $|a|<0.9$ are squeezed between the curves for $a=-0.9,0.9$. At larger inhomogeneities, $R / L=1$ (Fig. 12), one can still see the wellpronounced QSE oscillations, but the average is already noticeably shifted from zero as it should be at larger $R / L$ (cf. Fig. 9).

Note that the height of the first peak is always given by Eq. (43) and can be quite large when $a \rightarrow-1$ ("parallel" walls). At $a \rightarrow-1$ the interwall correlation compensates almost completely for dephasing caused by scattering from individual wall inhomogeneities. In this case, if the wall scattering is the only relaxation mechanism (ballistic transport), the lateral mean free path becomes infinitely large even if both walls are rough! In Figs. 11 and 12 the height of the first peak for $a=-0.9$ is 9 and the peak does not fit into the figures. For positive values of the interwall amplitude $a$ ("antiparallel" walls), the second peak has the largest amplitude, while the first (negative) one, which is given by Eq. (43), has a smaller amplitude.

In conclusion, the possible correlation of random inhomogeneities from the opposite walls provides a nontrivial insight into the mode coupling. On the other hand, measurements of the dependence of the transport coefficients on the film thickness or particle energy can provide unique information on the interwall correlations since, depending on the situation, the effect of interwall correlations can be constructive, destructive, or oscillating. The shift of oscillations gives the information on both the strength and sign of the interwall correlations.

\section{G. Scattering by multiscale inhomogeneities}

Above we studied the systems with random inhomogeneities of a single, well-defined spatial scale (correlation radius) $R$. In the case of single-scale inhomogeneities, such as 


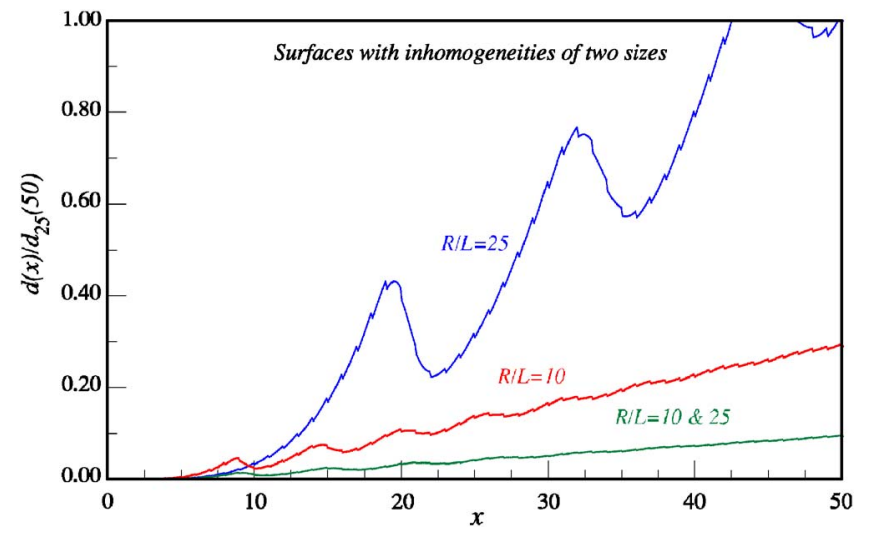

FIG. 13. (Color online) Diffusion coefficient for Gaussian inhomogeneities with $R / L=10, R / L=25$ and with the sum of inhomogeneities of both sizes. The curves are labeled by the value of $R / L$. All three curves are normalized by the value of $d(R / L=25 ; x=50)$.

inhomogeneities with a Gaussian or exponential power spectrum, the mode coupling channels at large $R$ open one by one at definite values of the film thickness (35) leading to largescale QSE oscillations of the transport coefficients. In the opposite case of the power spectrum with inhomogeneities of all sizes, such as slowly decaying power-law power spectrum with a low index, the mode coupling is always robust, leading to the disappearance of the large-scale oscillations and the restoration of the standard sawtooth QSE. It is interesting to investigate the behavior of QSE in an intermediate situation in system with inhomogeneities of few distinct scales. Figure 13 presents the data for the diffusion coefficient for a film with Gaussian surface inhomogeneities of three types: the inhomogeneities with $R / L=25, R / L=10$, and the inhomogeneities with a combination of both sizes (the sum of the corresponding power spectra). All three curves are normalized by the same value $d(R / L=25 ; x=50)$ and are labeled by the value of $R / L$. As one can easily see, the combining the inhomogeneities with two correlation sizes does not lead to a mechanical mixture of the individual oscillations but results in smoothing, shifting, and rescaling of the oscillations. Adding several more scales leads simply to a disappearance of the QSE oscillations. At present it is not clear yet the combination of how many scales are necessary for the restoration of the sawtooth behavior.

\section{CONCLUSIONS}

In summary, we analyzed the mode coupling and its effect on QSE in transport in high quality quasi-2D quantum systems $(R / L \gg 1)$ with various types of surface, thickness, and bulk fluctuations. Here are the conclusions:

(i) Though the transport equations and mode coupling effects for systems with bulk and interface fluctuations look almost identical, QSE in such systems is not the same. The appearance of large-scale oscillations of the transport coefficients requires not only the opening of mode coupling channels at distinct values of film thickness (35) and (36), as it happens in both surface- and bulk-driven systems, but also the predominant role of modes with low quantum numbers (grazing particles). The latter requirement is routine for surface scattering but is not fulfilled for bulk fluctuations of a general form. Only if the fluctuations in the bulk do not depend on the coordinate across the film, Eq. (25), the transport coefficients manifest the same large-scale oscillations as in the case of surface scattering. This explains a huge difference in QSE in high-quality films with bulk and surface scattering.

(ii) One of the most striking conclusions concerns the contributions from different modes in high-quality samples in the case of surface scattering by large inhomogeneities, $R / L \gg 1$. Without mode coupling, the contributions from a mode with quantum number $j$ would be proportional ${ }^{17}$ to $1 / j^{4}$ and higher modes would have been almost irrelevant. These higher modes contribute to transport only because of the mode coupling. This conclusion is of little interest for small-size defects $R / L \ll 1$ since in such systems the scattering-driven mode coupling is always robust and the contribution from the higher modes is important.

In high-quality films $R / L \gg 1$, the mode-coupling transitions switch on one by one at the values of the film thickness (35) and (36). Thus, the higher modes become important also one by one, only after the corresponding mode coupling channel is turned on, Fig. 6. After the mode coupling turns on, the contribution of the higher modes is much higher than one usually assumes and the description that singles out the grazing particles becomes wrong.

(iii) The consecutive opening of the mode coupling channels in high-quality films at distinct values of the film thickness, Eqs. (35) and (36), which leads to giant QSE oscillations, is a very robust effect that is not very sensitive to the nature of (quasi-)particles and the form of their spectrum. As a result, the effect can be observed in a wide variety of quantized systems such as metal or semiconductor films, quantum wires, ultranarrow channels, optical fibers, etc.

(iv) An interesting manifestation of the coupling effects in high-quality films is related to possible correlation between random inhomogeneities from the opposite walls. The interference of scattering from the opposite walls changes its sign from constructive to destructive depending on the parity of the sum of mode quantum numbers and, therefore, provides contributions of the opposite signs for intramode and mode coupling channels. In some cases ("parallel" walls) the opening of the mode coupling channel can be responsible for the cutoff for the mean free path for grazing particles which would be nearly divergent otherwise. The presence of interwall correlations can help to distinguish films with surface and thickness fluctuations. The shift of conductivity or diffusion oscillations provides the information on both the sign and strength of interwall correlations.

(v) The presence of multiscale inhomogeneities with several distinct correlation radii $R$ leads, instead of a mechanical mixture of individual QSE patterns, to shifting and smoothing of the QSE oscillations of the transport coefficients that are inherent to high-quality films with a single-scale roughness.

(vi) The results can lead to unique, nondestructive ways of studying the quality of the high quality surfaces, including the buried surfaces and interfaces, by measuring the lateral conductivity or diffusion. This is especially valuable for 
high-quality surfaces with large-scale inhomogeneities for which the usual scanning techniques can become problematic because of very large scanning areas.

The results of the paper can be applied to particles in a wide range of quantum quasi-2D systems. The results can also be cautiously extended even to quasi-1D systems up to the point when the strong localization effects render transport calculations meaningless (see review $^{30}$ and references therein).

\section{ACKNOWLEDGMENTS}

The work was supported by NSF Grant No. DMR0077266.
${ }^{1}$ P. A. Lee and T. V. Ramakrishnam, Rev. Mod. Phys. 57, 287 (1985); Mesoscopic Phenomena in Solids, edited by B. L. Altshuler, P. A. Lee, and R. A. Webb (Elsevier, Amsterdam, 1991); Scattering and Localization of Classical Waves in Random Media, edited by P. Sheng (World Scientific, Singapore, 1990).

${ }^{2}$ K. A. Muttalib, J.-L. Pichard, and A. D. Stone, Phys. Rev. Lett. 59, 2475 (1985); C. W. J. Beenakker, Rev. Mod. Phys. 69, 731 (1997).

${ }^{3}$ J. A. Sanchez-Gil, V. Freilikher, I. Yurkevich, and A. A. Maradudin, Phys. Rev. Lett. 80, 948 (1998).

${ }^{4}$ A. E. Meyerovich and A. Stepaniants, Phys. Rev. B 60, 9129 (1999).

${ }^{5}$ A. E. Meyerovich and I. V. Ponomarev, Phys. Rev. B 65, 155413 (2002).

${ }^{6}$ M. Jalochowski, M. Hoffmann, and E. Bauer, Phys. Rev. Lett. 76, 4227 (1996); Phys. Rev. B 51, 7231 (1995); L. A. Kuzik, Yu. E. Petrov, F. A. Pudonin, and V. A. Yakovlev, JETP 78, 114 (1994); G. M. Mikhailov, I. V. Malikov, and A. V. Chernykh, JETP Lett. 66, 725 (1997).

${ }^{7}$ M. Jalochowski, E. Bauer, H. Knoppe, and G. Lilienkamp, Phys. Rev. B 45, 13607 (1992); M. Jalochowski, M. Hoffmann, and E. Bauer, ibid. 51, 7231 (1995); H. Sakaki, T. Noda, K. Hirakawa, M. Tanaka, and T. Matsusue, Appl. Phys. Lett. 51, 1934 (1987); L.-W. Tu, G. K. Wong, and J. B. Ketterson, ibid. 55, 1327 (1989).

${ }^{8}$ J. J. Paggel, T. Miller, and T. C. Chang, Science 283, 1709 (1999); D. A. Evans, M. Alonso, R. Cimino, and K. Horn, Phys. Rev. Lett. 70, 3483 (1993); J. E. Ortega, F. J. Himpsel, G. J. Mankey, and R. F. Willis, Phys. Rev. B 47, 1540 (1993).

${ }^{9}$ J. J. Paggel, T. Miller, and T. C. Chiang, Phys. Rev. Lett. 81, 5632 (1998); F. Patthey and W.-D. Schneider, Phys. Rev. B 50, 17560 (1994); M. Schmid, W. Hebenstreit, P. Varga, and S. Crampin, Phys. Rev. Lett. 76, 2298 (1996).

${ }^{10} \mathrm{~S}$. Andrieu, C. Chatelain, M. Lemine, B. Berche, and Ph. Bauer, Phys. Rev. Lett. 86, 3883 (2001).

${ }^{11}$ I. B. Altfeder, D. M. Chen, and K. A. Matveev, Phys. Rev. Lett. 80, 4895 (1998); I. B. Altfeder, K. A. Matveev, and D. M. Chen, ibid. 78, 2815 (1997).

${ }^{12}$ V. B. Sandomirskii, Sov. Phys. JETP 25, 101 (1967) [Zh. Eksp. Teor. Fiz. 52, 158 (1968)].
${ }^{13}$ N. Trivedi and N. W. Ashcroft, Phys. Rev. B 38, 12298 (1988).

${ }^{14}$ S. L. Phillipson, A. M. Guenault, S. N. Fisher, G. R. Pickett, and P. J. Y. Thibault, Nature (London) 395, 578 (1998); P. A. Reeves, A. M. Guenault, S. N. Fisher, G. R. Pickett, and G. Tvalashvili, Physica B 284-288, 319 (2000).

${ }^{15}$ A. E. Meyerovich and R. Jochemsen, J. Low Temp. Phys. 126, 193 (2001)

${ }^{16}$ V. V. Nesvizhevsky, H. G. Borner, A. K. Petoukhov, H. Abele, S. Baessler, F. J. Ruess, Th. Stoferle, A. Westphal, A. M. Gargansky, G. A. Petrov, and A. V. Strelkov, Nature (London) 415, 297 (2002); V. V. Nesvizhevsky, H. G. Borner, A. M. Garganski, A. K. Petoukhov, G. A. Petrov, H. Abele, S. Baessler, G. Divkovic, F. J. Ruess, T. Stoferle, A. Westphal, A. V. Strelkov, K. V. Protasov, and A. Yu. Voronin, Phys. Rev. D 67, 102002 (2003).

${ }^{17}$ A. E. Meyerovich and A. Stepaniants, Phys. Rev. B 58, 13242 (1998).

${ }^{18}$ A. E. Meyerovich and A. Stepaniants, J. Phys.: Condens. Matter 12, 5575 (2000).

${ }^{19}$ Z. Tesanovic, M. V. Jaric, and S. Maekawa, Phys. Rev. Lett. 57, 2760 (1986).

${ }^{20}$ G. Fishman and D. Calecki, Phys. Rev. Lett. 62, 1302 (1989).

${ }^{21}$ A. Kawabata, J. Phys. Soc. Jpn. 62, 3988 (1993).

${ }^{22}$ A. E. Meyerovich and S. Stepaniants, Phys. Rev. Lett. 73, 316 (1994); Phys. Rev. B 51, 17116 (1995); J. Phys.: Condens. Matter 9, 4157 (1997).

${ }^{23}$ N. M. Makarov, A. V. Moroz, and V. A. Yampol'skii, Phys. Rev. B 52, 6087 (1995).

${ }^{24}$ A. E. Meyerovich and A. Stepaniants, Aust. J. Phys. 53, 53 (2000).

${ }^{25}$ J. A. Ogilvy, Theory of Wave Scattering from Random Surfaces (Adam Hilger, Bristol, 1991).

${ }^{26}$ R. M. Feenstra, D. A. Collins, D. Z. Y. Ting, M. W. Wang, and T. C. McGill, Phys. Rev. Lett. 72, 2749 (1994).

${ }^{27}$ G. Fishman and D. Calecki, Phys. Rev. B 43, 11581 (1991).

${ }^{28}$ G. Palasantzas and J. Barnas, Phys. Rev. B 56, 7726 (1997); G. Palasantzas, Y.-P. Zhao, G.-C. Wang, T.-M. Lu, J. Barnas, and J. Th. M. De Hosson, ibid. 61, 11109 (2000).

${ }^{29}$ I. V. Ponomarev, L. I. Deych, and A. A. Lisyansky, Appl. Phys. Lett. 85, 2496 (2004).

${ }^{30}$ F. M. Izrailev and N. M. Makarov, J. Phys. A 38, 10613 (2005). 